\title{
Concentration Fluctuations in a Polymer Solution under a Temperature Gradient
}

\author{
W. B. Li, ${ }^{1}$ K. J. Zhang, ${ }^{1}$ J. V. Sengers, ${ }^{1,2}$ R. W. Gammon, ${ }^{1}$ and J. M. Ortiz de Zárate ${ }^{3}$ \\ ${ }^{1}$ Institute for Physical Science and Technology, University of Maryland, College Park, Maryland 20742 \\ ${ }^{2}$ Department of Chemical Engineering, University of Maryland, College Park, Maryland 20742 \\ ${ }^{3}$ Facultad de Ciencias Físicas, Universidad Complutense, 28040 Madrid, Spain
}

(Received 15 June 1998)

We have performed small-angle Rayleigh light-scattering measurements in polymer solutions under various externally applied temperature gradients. Our experiments confirm the existence of an enhancement of the concentration fluctuations. The nonequilibrium concentration fluctuations are proportional to $(\nabla T)^{2} / k^{4}$, where $\nabla T$ is the applied temperature gradient, and $k$ is the wave number of the fluctuations. The measured strengths of the nonequilibrium fluctuations in the dilute and semidilute concentration regime agree with the strengths calculated from fluctuating hydrodynamics. [S0031-9007(98)08007-7]

PACS numbers: $61.25 . \mathrm{Hq}, 05.70 . \mathrm{Ln}, 66.10 . \mathrm{Cb}$

During the past decade, considerable theoretical and experimental effort has been devoted to the study of fluctuations in fluids in nonequilibrium steady states, in particular, when subjected to a constant temperature gradient $\nabla T$. It is now known that the correlation functions become spatially long ranged [1-3], because microscopic detailed balance is violated. These nonequilibrium correlation functions can be observed by small-angle Rayleigh light scattering. Experiments have been carried out with simple fluids, in which case excellent agreement with the theory has been found [4,5].

In fluid mixtures, the situation is more complicated because the temperature gradient induces a concentration gradient through the Soret effect. Hence, in addition to nonequilibrium entropy and viscous fluctuations, which are also present in one-component fluids, nonequilibrium concentration fluctuations are present in mixtures [6]. All of these nonequilibrium fluctuations have intensities proportional to $(\nabla T)^{2} / k^{4}$, where $k$ is the wave number of the fluctuations. The existence of these nonequilibrium fluctuations and their dependence on $(\nabla T)^{2} / k^{4}$ have been checked experimentally, but the quantitative values of the observed strengths of the nonequilibrium concentration fluctuations were not in agreement with the theory $[5,7,8]$.

Dilute polymer solutions appear to be a more suitable system to check the theory for nonequilibrium concentration fluctuations, because the expression for the dynamic correlation function $C(k, t)$ as a function of time is simpler. When the polymer concentration is below the overlap concentration, viscoelastic and entanglement effects are negligible, and ordinary fluctuating hydrodynamics should be applicable to describe the nonequilibrium fluctuations. Furthermore, the collective mass-diffusion coefficient is so small and the Rayleigh ratio so large that, in practice, the concentration fluctuations are dominant. Therefore, for polymer solutions at sufficiently low con- centrations, the equations in Refs. [5,6] can be simplified, yielding the following for fluctuations with a wave vector perpendicular to $\nabla T$ :

$$
C(k, t)=C_{0}\left[1+A_{c}(\nabla T, k)\right] e^{-D k^{2} t},
$$

where $C_{0}$ is the ratio of the signal to the local oscillator background, which corresponds to the amplitude of the experimental equilibrium correlation function; $D$ is the collective mass-diffusion coefficient, and $A_{c}$ is the nonequilibrium enhancement. $A_{c}$ may be expressed as

$$
A_{c}(\nabla T, k)=A_{c}^{*}(w) \frac{(\nabla T)^{2}}{k^{4}},
$$

where the strength of the enhancement, $A_{c}^{*}(w)$, is given by

$$
A_{c}^{*}(w)=\frac{[w(1-w)]^{2} S_{T}^{2}}{\nu D}\left(\frac{\partial \mu}{\partial w}\right)_{p, T} .
$$

Here, $w$ is the concentration expressed as a mass fraction of the polymer, $\nu$ is the zero-shear kinematic viscosity of the solution, and $\mu=\mu_{1}-\mu_{2}$ is the difference between the chemical potentials per unit mass of the two components of the mixture. The Soret coefficient $S_{T}$ specifies the ratio between temperature and concentration gradients and is defined through the steady-state phenomenological equation:

$$
\nabla w=-S_{T} w(1-w) \nabla T .
$$

It is worth mentioning that in the limit $\nabla T \rightarrow 0$ the set of Eqs. (1)-(3) reduces to the well-known expression for the equilibrium time correlation function of a polymer solution. This equilibrium expression is the basis of the dynamic light-scattering method widely employed for the measurement of diffusion coefficients in polymer solutions. It is also interesting to note that Eqs. (1) -(3) can be obtained from the theory of Schmitz for the nonequilibrium concentration fluctuations in a colloidal suspension [9] in the limit of small colloidal particle size. 
Some limitations apply to Eqs. (1)-(3) to describe the concentration fluctuations in a nonequilibrium polymer solution. Specifically, when the polymer concentration increases, due to the interaction between polymer chains, viscoelastic and entanglement effects are no longer negligible. In addition, for extremely small $k$, a saturation in the nonequilibrium enhancement appears due to gravity and boundary effects [10], but this last effect is not relevant for the wave numbers probed in the present experiments.

The goal of this Letter is to report recent results obtained in our laboratory, which confirm the ability of Eqs. (1)-(3) to describe the concentration fluctuations in a polymer solution subjected to a stationary temperature gradient. To perform the experiments, we have chosen the polymer/solvent system polystyrene/toluene, because this system has been widely investigated, and reliable information for the thermophysical properties is available. The toluene was a Fisher certified reagent with purity $99.9 \%$. The polystyrene was obtained from the Tosoh Corporation (Japan) and was characterized by the manufacturer with $\bar{M}_{W}=96400$ and polydispersivity 1.01 . From Ref. [11], the radius of gyration and overlap concentration for this polymer in toluene solutions may be estimated as $R_{g} \approx 11 \mathrm{~nm}$ and $w^{*} \approx 3.1 \%$. Five polystyrene/toluene solutions with concentrations below $w^{*}$ and one slightly above $w^{*}$ were prepared gravimetrically and were agitated for at least one hour before use. The polymer solutions were introduced in the light-scattering cell through $0.5 \mu \mathrm{m}$ millipore Millex-LCR Teflon HPLC filters. At the time when the cell was being filled, a portion of the polymer solution prepared in the same bottle was introduced in a beam-bending cell [8], enabling us to measure $D$ and $S_{T}$ of the solutions [12]. The results obtained with this beam-bending technique will be used for direct comparison with the results obtained here from light scattering.

A complete description of the small-angle lightscattering setup may be found elsewhere [5]. The fluidsample cell is a cylindrical layer of thickness $0.118 \pm$ $0.005 \mathrm{~cm}$ bound between two copper plates and a glass ring sealed against the top and the bottom plates with indium O-rings. The copper plates are furnished with parallel optical windows and independent temperaturecontrol systems, able to maintain different temperatures at each plate. The temperatures are measured by thermistors placed inside the copper plates. When the control system is working, the temperatures of the plates are stable within $\pm 10 \mathrm{mK}$. The incident helium-neon laser beam, with an output power of $6 \mathrm{~mW}$, propagates through the optical windows, parallel to the vertical temperature gradient to avoid beam bending. The scattering angle is selected with a small collecting pinhole $(500 \mu \mathrm{m})$ located $197 \mathrm{~mm}$ after the cell at a few millimeters from the transmitted beam. This geometry selects fluctuation wave vectors essentially perpendicular to $\nabla T$. The distance between the transmitted beam and the scattering collection pinhole was measured with a Vernier micrometer scale, providing an accurate value for the mean scattering angle $\theta$ (the refraction in the sapphire windows has to be taken into account in the calculation of $\theta$ ). The accuracy in the determination of the wave vector $k$ in our experiments is $1.5 \%$.

The nonequilibrium enhancement given by Eq. (2) becomes measurable only at small scattering angles because of its dependence on $k^{-4}$. In the present work, at least four scattering angles were employed for each solution between $0.4^{\circ}$ and $0.9^{\circ}$, corresponding to wave numbers ranging between 900 and $2000 \mathrm{~cm}^{-1}$. At these small scattering angles, the scattered light from window inner surfaces is dominant, assuring that the measurements are in the heterodyne regime. The light exiting through the collecting pinhole is focused onto a photomultiplier and analyzed with an ALV-5000 multiple tau correlator.

The experiments started by measuring the equilibrium time correlation function with both plates at $25^{\circ} \mathrm{C}$. Subsequently, the temperatures of the top and the bottom plates were changed symmetrically, the average cell temperature remaining at $25^{\circ} \mathrm{C}$, while heating from above to avoid convection. The maximum temperature difference employed was $4.1^{\circ} \mathrm{C}$, which corresponds to a maximum temperature gradient of $34.6 \mathrm{~K} \mathrm{~cm}^{-1}$. The variation in the thermophysical properties of the solution is negligible in this small temperature interval, and, in all calculations, values corresponding to $25^{\circ} \mathrm{C}$ were used. For each solution and wave number, two to six values of $\nabla T$ were employed, and, for each $\nabla T$, four to ten correlograms were measured, each lasting at least $30 \mathrm{~min}$. The correlograms, in the range from $10^{-2}$ to $10 \mathrm{~s}$, were fitted to a single exponential in accordance with Eq. (1). In all cases, both in equilibrium and in nonequilibrium states, very good fits were obtained, yielding values for the decay rate $D k^{2}$ and for the amplitude $C_{0}\left[1+A_{c}(\nabla T, k)\right]$ of the exponential. Average values for these parameters were calculated for each concentration $w$, at each wave number $k$, and each temperature gradient $\nabla T$ (including zero). The intensity of the fluctuations in each run was calculated by multiplying the amplitude of the correlation function by the average count rate. The amplitude of the equilibrium correlation function had values $10^{-4}$ to $10^{-3}$. The average detected photon count rate was 0.3 to $2.5 \mathrm{MHz}$.

From the experimental decay rates and the known values of the scattering wave number $k$, the diffusion coefficient can be calculated. The experimental values of the diffusion coefficient are displayed in Fig. 1 as a function of the concentration $c$ in $\mathrm{g} \mathrm{cm}^{-3}$. As shown in Fig. 1, the agreement of the present results with the values obtained from beam bending [12] is satisfactory.

As usual, the collective mass-diffusion coefficient in the dilute regime was fitted to the linear relationship

$$
D(c)=D_{0}\left(1+k_{D} c\right),
$$


where $D_{0}$ is the diffusion coefficient at infinite dilution, and $k_{D}$ is the diffusional virial coefficient. We obtain, from a least-squares analysis of our data, the values $D_{0}=(4.9 \pm 0.2) \times 10^{-7} \mathrm{~cm}^{2} \mathrm{~s}^{-1}$ and $k_{D}=21 \pm$ $2 \mathrm{~cm}^{3} \mathrm{~g}^{-1}$. A review of literature values for these coefficients [13] yields $D_{0}=(4.9 \pm 0.2) \times 10^{-7} \mathrm{~cm}^{2} \mathrm{~s}^{-1}$ and $k_{D}=22 \pm 5 \mathrm{~cm}^{3} \mathrm{~g}^{-1}$, in excellent agreement with our experimental results.

The intensity of the fluctuations shows a dramatic increase with increasing values of the temperature gradient. By comparing the intensities of the equilibrium and the nonequilibrium concentration fluctuations, the enhancement $A_{c}(\nabla T, k)$ was determined. First of all, the expected dependence on $(\nabla T)^{2} / k^{4}$ was checked. As examples, we show in Fig. 2 the enhancements measured at different $\nabla T$ and $k$ as a function of $(\nabla T)^{2} / k^{4}$, for $w=4.00 \%$ and $w=0.50 \%$. The experimentally observed enhancement of the concentration fluctuations indeed varies linearly with $(\nabla T)^{2} / k^{4}$.

From the slope of the fitted lines, such as the ones in Fig. 2, the experimental values for the strength of the enhancement, $A_{c}^{*}(w)$, can be deduced. The values obtained may be compared with those calculated from Eq. (3) and the known thermophysical properties of the solutions. To do so, we need, in addition to the diffusion coefficient $D$, values for the kinematic viscosity $\nu$, the osmotic compressibility $(\partial \mu / \partial w)_{P, T}$, and the Soret coefficient. Values for $\nu$ and $(\partial \mu / \partial w)_{P, T}$ were obtained from the literature [11,14]. As mentioned earlier, $S_{T}$ of our solutions was measured by Zhang et al. [12], yielding values in good agreement with data obtained by Köhler et al. [15]. Further details will be presented in a subsequent publication. Figure 3 shows the experimentally measured values for $A_{c}^{*}$ compared with the values calculated from

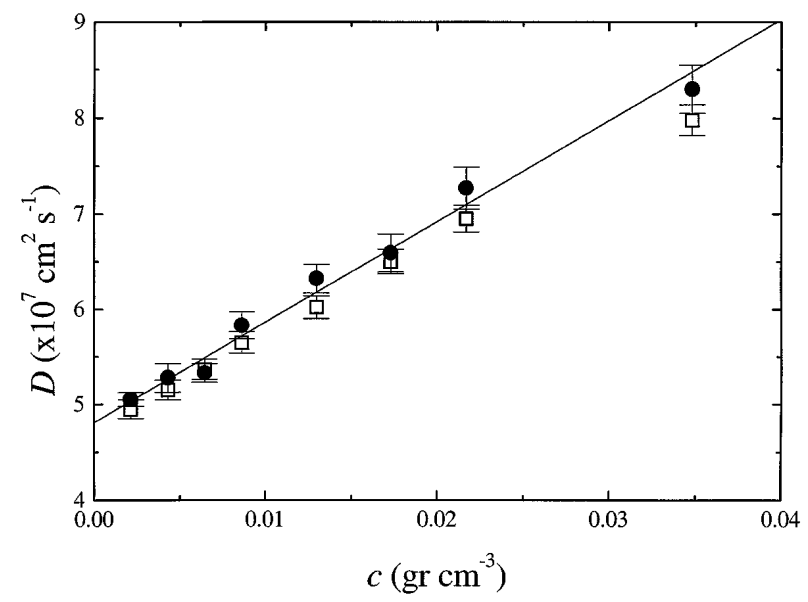

FIG. 1. Concentration dependence of the collective massdiffusion coefficient $D$ in polystyrene/toluene solutions. : measured in this work by equilibrium and nonequilibrium Rayleigh light scattering. $\square$ : measured by Zhang et al. with a beam-bending technique [12]. The straight line is a fit to the present data.
Eq. (3). Good agreement between theory and experiment is obtained both in the dilute and in the semidilute regime.

In conclusion, we have demonstrated that long-range concentration fluctuations in polymer solutions subjected to a stationary temperature gradient can be observed by small-angle nonequilibrium Rayleigh light scattering. Similar to the nonequilibrium fluctuations observed in a liquid mixture [7], the nonequilibrium enhancement of the concentration fluctuations has been found to be proportional to $(\nabla T)^{2} / k^{4}$. However, unlike the case of a liquid mixture, where the strength of the observed nonequilibrium fluctuations appeared to be inconsistent with the observed Soret effect $[5,7,8]$, we have now found close agreement between experiment and theory for the strength $A_{c}^{*}$ of the nonequilibrium concentration fluctuations induced by a stationary temperature gradient. This supports the validity of nonequilibrium fluctuating hydrodynamics and the feasibility of small-angle Rayleigh light scattering in the study of thermal nonequilibrium steady states in polymer solutions. Further theoretical and experimental work will be needed to understand nonequilibrium concentration fluctuations in polymer solutions at higher concentrations.

Our present results complement considerable theoretical and experimental progress recently made in understanding the dynamics of concentration fluctuations of polymer solutions under shear flow. It has been known for some time that concentration fluctuations in semidilute polymer solutions subjected to shear flow are dramatically enhanced [16], as we have found here when a temperature gradient is applied.

We thank S. C. Greer for helpful advice concerning the characterization of the polymer sample and W. Köhler

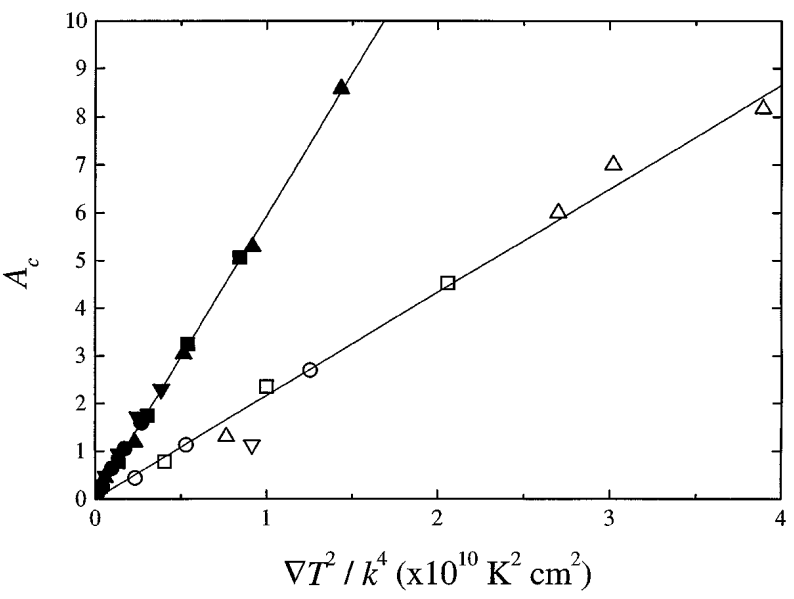

FIG. 2. The nonequilibrium enhancement $A_{c}$ of the concentration fluctuations in dilute and semidilute polystyrene/toluene solutions at $25^{\circ} \mathrm{C}$ as a function of $(\nabla T)^{2} / k^{4}$. The solid symbols correspond to the concentration $w=4.00 \%$ and the open symbols to $w=0.50 \%$. Different symbol shapes correspond to different values of $k$. The solid lines are linear fits to the experimental data for each concentration. 


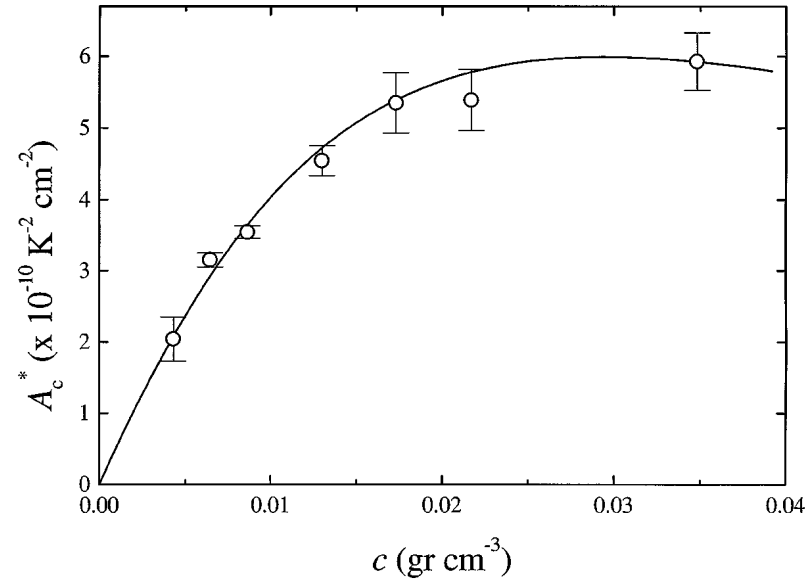

FIG. 3. The strength $A_{c}^{*}$ of the nonequilibrium concentration fluctuations as a function of the polymer concentration. The symbols represent the experimental data and the curve represents the values calculated from Eq. (3).

for valuable information for the Soret coefficient. This research is supported by the National Science Foundation, Grant No. CHE-9805260. J. M. O.Z. was funded by the Spanish Department of Education during his postdoctoral stage at Maryland, where the work was done.

[1] T.R. Kirkpatrick, E. G.D. Cohen, and J.R. Dorfman, Phys. Rev. A 26, 995 (1982).

[2] G. Grinstein, D.-H. Lee, and S. Sachdev, Phys. Rev. Lett. 64, 1927 (1990).

[3] J. R. Dorfman, T. R. Kirkpatrick, and J. V. Sengers, Annu. Rev. Phys. Chem. 45, 213 (1994).
[4] P. N. Segrè, R. W. Gammon, J. V. Sengers, and B. M. Law, Phys. Rev. A 45, 714 (1992).

[5] W. B. Li, P. N. Segrè, R. W. Gammon, and J. V. Sengers, Physica (Amsterdam) 204A, 399 (1994).

[6] B. M. Law and J. C. Nieuwoudt, Phys. Rev. A 40, 3880 (1989); R. M. Velasco and L. S. García-Colín, J. Phys. A 24, 1007 (1991).

[7] P. N. Segrè, R. W. Gammon, and J. V. Sengers, Phys. Rev. E 47, 1026 (1993).

[8] K. J. Zhang, M.E. Briggs, R.W. Gammon, and J. V. Sengers, J. Chem. Phys. 104, 6881 (1996).

[9] R. Schmitz, Physica (Amsterdam) 206A, 25 (1994).

[10] P. N. Segrè, R. Schmitz, and J. V. Sengers, Physica (Amsterdam) 195A, 31 (1993); A. Vailati and M. Giglio, Phys. Rev. Lett. 77, 1484 (1996).

[11] I. Noda, Y. Higo, N. Ueno, and T. Fujimoto, Macromolecules 17, 1055 (1984); Y. Higo, N. Ueno, and I. Noda, Polym. J. 15, 367 (1983).

[12] K. J. Zhang, M.E. Briggs, R. W. Gammon, J. V. Sengers, and J.F. Douglas (to be published).

[13] The literature on the diffusion coefficient and hydrodynamic interaction for polystyrene/toluene has been reviewed in the thesis by W.B. Li [Ph.D. dissertation, University of Maryland, College Park, MD, 1996, Table 6.3, p. 223].

[14] Y. Takakashi, Y. Isono, I. Noda, and M. Nagasawa, Macromolecules 18, 1002 (1985); L. A. Papazian, Polymer 10, 399 (1969).

[15] W. Köhler, C. Rosenauer, and P. Rossmanith, Int. J. Thermophys. 16, 11 (1995).

[16] X.L. Wu, D. J. Pine, and P. K. Dixon, Phys. Rev. Lett. 66, 2408 (1991); S. T. Milner, Phys. Rev. Lett. 66, 1477 (1991); A. Onuki, Phys. Rev. Lett. 62, 2472 (1989). 